\title{
Cost impact of procalcitonin-guided decision making on duration of antibiotic therapy for suspected early-onset sepsis in neonates
}

A. J. L. M. Geraerds ${ }^{1 *+} \mathbb{D}$, Wendy van Herk ${ }^{2 \dagger}$, Martin Stocker ${ }^{3}$, Salhab el Helou ${ }^{4}$, Sourabh Dutta ${ }^{4}$, Matteo S. Fontana ${ }^{3}$, Frank A. B. A. Schuerman ${ }^{5}$, Rita K. van den Tooren-de Groot ${ }^{6}$, Jantien Wieringa ${ }^{6}$, Jan Janota7,8, Laura H. van der Meer-Kappelle ${ }^{9}$, Rob Moonen ${ }^{10}$, Sintha D. Sie ${ }^{11}$, Esther de Vries ${ }^{12}$, Albertine E. Donker ${ }^{13}$, Urs Zimmerman ${ }^{14}$, Luregn J. Schlapbach ${ }^{15,16,17}$, Amerik C. de Mol ${ }^{18}$, Angelique Hoffman-Haringsma ${ }^{19}$, Madan Roy ${ }^{20}$, Maren Tomaske ${ }^{21}$, René F. Kornelisse ${ }^{22}$, Juliette van Gijsel ${ }^{23}$, Eline G. Visser², Annemarie M. C. van Rossum² and Suzanne Polinder ${ }^{1}$

\begin{abstract}
Backgrounds: The large, international, randomized controlled NeoPInS trial showed that procalcitonin (PCT)-guided decision making was superior to standard care in reducing the duration of antibiotic therapy and hospitalization in neonates suspected of early-onset sepsis (EOS), without increased adverse events. This study aimed to perform a costminimization study of the NeoPInS trial, comparing health care costs of standard care and PCT-guided decision making based on the NeoPInS algorithm, and to analyze subgroups based on country, risk category and gestational age.

Methods: Data from the NeoPInS trial in neonates born after 34 weeks of gestational age with suspected EOS in the first $72 \mathrm{~h}$ of life requiring antibiotic therapy were used. We performed a cost-minimization study of health care costs, comparing standard care to PCT-guided decision making.

Results: In total, 1489 neonates were included in the study, of which 754 were treated according to PCT-guided decision making and 735 received standard care. Mean health care costs of PCT-guided decision making were not significantly different from costs of standard care (€3649 vs. €3616). Considering subgroups, we found a significant reduction in health care costs of PCT-guided decision making for risk category'infection unlikely' and for gestational age $\geq 37$ weeks in the Netherlands, Switzerland and the Czech Republic, and for gestational age $<37$ weeks in the Czech Republic.

Conclusions: Health care costs of PCT-guided decision making of term and late-preterm neonates with suspected EOS are not significantly different from costs of standard care. Significant cost reduction was found for risk category 'infection unlikely', and is affected by both the price of PCT-testing and (prolonged) hospitalization due to SAEs.
\end{abstract}

\footnotetext{
*Correspondence: a.geraerds@erasmusmc.n

${ }^{\dagger}$ A. J .L. M. Geraerds and Wendy van Herk have contributed equally to this work

${ }^{1}$ Department of Public Health, Erasmus MC, University Medical Center Rotterdam, P.O. Box 2040, 3000 CA Rotterdam, The Netherlands Full list of author information is available at the end of the article
}

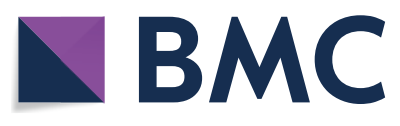

(c) The Author(s) 2021. Open Access This article is licensed under a Creative Commons Attribution 4.0 International License, which permits use, sharing, adaptation, distribution and reproduction in any medium or format, as long as you give appropriate credit to the original author(s) and the source, provide a link to the Creative Commons licence, and indicate if changes were made. The images or other third party material in this article are included in the article's Creative Commons licence, unless indicated otherwise in a credit line to the material. If material is not included in the article's Creative Commons licence and your intended use is not permitted by statutory regulation or exceeds the permitted use, you will need to obtain permission directly from the copyright holder. To view a copy of this licence, visit http://creativecommons.org/licenses/by/4.0/. The Creative Commons Public Domain Dedication waiver (http://creativeco mmons.org/publicdomain/zero/1.0/) applies to the data made available in this article, unless otherwise stated in a credit line to the data. 
Keywords: Neonates, Procalcitonin-guided decision making, Sepsis, Costs

\section{Background}

Early-onset sepsis (EOS) is one of the main causes for hospitalization in the first week of life. Annually, approximately $4-7 \%$ of term and late-preterm neonates in highincome countries are treated with intravenous antibiotics because of suspected EOS, whereas the prevalence of EOS is only $0.1 \%$ [1]. This implicates unnecessary antibiotic treatment in the majority of the treated neonates. Moreover, antibiotic treatment is associated with undesirable consequences, such as hospital admission, neonatal and parental discomfort, alterations in the neonatal microbiome and the use of health care resources, which puts a high demand on health care costs [2, 3].

One approach to improve the management of EOS is by shortening the duration of antibiotic treatment, using biomarker guidance. A successful biomarker-based strategy that was identified in previous studies is procalcitonin (PCT)-guided decision making [4-6]. In the NeoPInS study, PCT-guided decision making was found to significantly reduce the duration of antibiotic treatment, with unchanged outcome for adverse events [7]. Whether PCT-guided decision making is also cost-effective has not been evaluated yet. The main reason for concerns with respect to cost-effectiveness is the price of a PCT-test: a PCT-test is more expensive than a CRP-test. A previous patent on the PCT-test has expired, which has led to more market competition, and therefore a lower price for the PCT-test kit. This has resulted in lower costs of PCT-testing and might therefore result in PCT-guided decision making being more cost-effective.

There have been cost analyses of PCT-guided decision making in critically ill adult patients [8-10], and in patients with sepsis and lower respiratory tract infection (LRTI) $[11,12]$. Voermans et al. and Mewes et al. found that PCT-guided decision making led to a cost saving in both sepsis patients and LRTI patients, mainly due to a reduced duration of hospitalization [12], but also due to a reduction in antibiotic days, shorter duration of mechanical ventilation and fewer patients at risk for antibiotic resistant or C. difficile infection [11]. For critically ill patients, it was found that PCT-guided decision making led to significantly lower hospital costs and shorter duration of hospitalization $[8,9]$. However, Kip et al. found no significant difference in total health care-related costs [10].

To our knowledge, no study has investigated health care use and costs of PCT-guided decision making in children. This paper, based on the NeoPInS study, describes whether PCT-guided decision making for neonates with suspected EOS can safely reduce medical costs. In addition, it explores differences in costs between subgroups based on country, risk classification and gestational age.

\section{Methods \\ Study design}

This study explored the total direct medical costs of PCTguided decision making in neonates with suspected EOS from a hospital-based perspective. Assessment was based on the Neonatal PCT Intervention Study (NeoPInS), a randomized open controlled international multicenter intervention trial. Patients were enrolled in 18 hospitals, situated in the Netherlands $(n=11)$, Switzerland $(n=4)$, Canada $(n=2)$ and the Czech Republic $(n=1)$. Ethical approval of the protocol was obtained. Furthermore, written informed consent was obtained for all study participants. For more detailed information regarding the methods, study design and participants of the NeoPInS study, we refer to the original paper [7, 13]. The most important aspects are presented below.

\section{Participants}

Neonates born between May 21, 2009 and February 14, 2015 after 34 weeks of gestational age with suspected EOS in the first $72 \mathrm{~h}$ of life requiring antibiotic therapy were eligible for inclusion. This study only included neonates with no missing key variables. Therefore, we excluded 4 neonates from the PCT-guided decision-making group, and 17 neonates from the standard care group that were specified in the original paper [7].

\section{Procedures}

Within $12 \mathrm{~h}$ after start of therapy, all neonates were stratified into four risk categories, based on three elements: clinical symptoms, risk factors and conventional laboratory measurements (Additional file 1: Figure S1). The four categories were: category 1 (neonates with a proven infection with a positive blood culture), category 2 (neonates with high risk of infection due to clinical symptoms, risk factors and abnormal laboratory findings), category 3 (neonates with a possible risk of infection based on two out of three elements of the risk stratification) and category 4 (neonates where infection is unlikely, based on one out of three elements from the risk stratification). All neonates in categories 1 and 2 were given standard care according to local policy (a minimum of 7 days of antibiotic treatment). PCT-guided decision making was not applied since their high risk of infection would not allow shorter antibiotic treatment. Therefore, neonates in 
category 1 and 2 were excluded in this study. The antibiotic treatment of neonates in category 3 and 4 was based on either PCT-guided decision making (intervention) or standard care (based on local policy) (Additional file 1: Figure S1).

PCT-guided decision making was defined by a minimum treatment duration of $24 \mathrm{~h}$, where the decision to discontinue antibiotic treatment was based on the measured procalcitonin values: antibiotic therapy could be discontinued when two consecutive PCT values were within normal values according to the postpartum nomogram [7]. Physicians decided when a neonate was discharged from the hospital, and were at all times allowed to overrule the recommendation from the treatmentrelated algorithm based on for example clinical symptoms or other laboratory investigations.

\section{Resource data}

During the trial, data were retrieved from individual patient records in a case record form (CRF) by local investigators. A monitoring team performed $100 \%$ source data verification through onsite visits, in order to ensure data quality and completeness. However, due to unavailability of key information in some patient files, some missing values in key variables remained. Database access was restricted to the data management team until the end of the trial.

For all patients, follow-up information on the first month of life was obtained, regarding recurrence of infection, readmission to the hospital, additional courses of antibiotics and death. Information on serious adverse events (SAEs) (e.g., hyperbilirubinemia, feeding problems due to prematurity) was retrieved by monitoring.

\section{Cost study}

As no effect of PCT-guided decision making compared to standard care was found in mortality and morbidity [7], a cost minimization analysis was conducted. The cost minimization analysis comprised a direct comparison between total health care costs of PCT-guided decision making and standard care, from a health care perspective. All health care resources that were used in the hospital were registered in a CRF. Additional days of hospitalization and readmission due to SAEs were included in the total duration of hospitalization, and additional antibiotic treatment was included in the total duration of antibiotics treatment. Estimates of unit costs were based on the Dutch guideline prices, or were obtained from the website of the Dutch Health Insurance Board [14, 15]. The cost price of PCT-testing was calculated based on the micro-costing method. The mean of all prices of PCTtesting, which were retrieved from the participating hospitals, was used as the price per PCT-test. Total medical costs were calculated by multiplying volumes of health care resources with corresponding unit prices, and consisted of costs of hospital days, costs of antibiotics, costs of laboratory tests (CRP and PCT) and labor costs. Costs were calculated in the European currency (Euro), corrected for inflation for the year 2015. All prices included in this cost study are presented in Additional file 2 (Table S1). Discounting was not applied, since the followup period of the study comprised one month.

Unit prices for Switzerland $(\mathrm{CH})$, Canada $(\mathrm{CA})$ and the Czech Republic $(\mathrm{CZ})$ were based on the Dutch prices, corrected for the purchasing power parity (PPP) for the general domestic product (GDP). Subgroups based on country, age (gestational age $<37$ weeks or $\geq 37$ weeks) and risk category were compared to explore potential cost drivers.

\section{Sensitivity analysis}

Sensitivity analysis was performed by varying costs of PCT-testing, to study the influence of changes in pricing on the results. Costs of PCT-testing were examined using the minimum ( $\mathrm{NL} € 13.70$; $\mathrm{CH} € 17.50$; $\mathrm{CA} € 13.50$; $\mathrm{CZ} € 7.80$ ) and maximum prices (NL €33.90; $\mathrm{CH} € 43.40$; $C A € 33.40$; $C Z € 19.50)$. A second sensitivity analysis was performed by excluding additional days of hospitalization due to SAEs (e.g., hyperbilirubinemia/feeding problems), with the aim to provide insight in the effect of the SAEs on the total health care costs. The SAEs were deemed by the data safety and monitoring board as not-related to the study. A third sensitivity analysis was performed using the per-protocol population, in order to express the real potential of PCT-guided decision making. Neonates with protocol violations were excluded in the per-protocol analyses.

\section{Statistical analysis}

An intention-to-treat analysis was performed to express the real clinical situation of PCT-guided decision making. For clinical and demographical data, a comparison between groups for categorical variables was made using either the $\chi^{2}$ test or the Fisher exact test when appropriate. For continuous variables, nonparametric analysis was performed using the Mann-Whitney $U$ test. $P$ values $<0.05$ were considered to indicate statistical significance. Variables with multiple categories, such as country and delivery method, were compared using the KruskalWallis test.

Mean costs were reported in euro $(€)$ with the interquartile range (IQR). Differences in costs between the PCT-guided decision-making group and the standard care group were compared using Mann-Whitney $\mathrm{U}$ test, since costs were not normally distributed. Due to the exploratory nature of the subgroup analyses, no 
correction for multiplicity was applied. Analyses were performed using SPSS software package, version 25 (SPSS Inc., Chicago, Illinois, USA).

\section{Results \\ Study population}

Overall, 2440 neonates with suspected EOS were screened, of which 730 neonates were excluded for reasons described in the original study [7]. In this study, we excluded 21 more neonates due to missing information on duration of hospitalization and antibiotic treatment. Of the remaining 1689 neonates, 1489 were classified as risk category 'infection possible' or 'infection unlikely, and randomly assigned to either PCT-guided decision making $(n=754)$ or standard care $(n=735)$ (Fig. 1$)$. No significant differences in baseline characteristics were found between the PCT-guided decision-making group and the standard care group (Table 1). For the sensitivity analyses in the per protocol population, neonates with protocol violations were excluded. This resulted in the inclusion of 678 neonates in the per protocol analysis of the PCT-guided decision-making group, and 606 neonates in the standard care group.

\section{Duration of hospitalization and antibiotic treatment}

In all countries combined and individually, neonates treated with PCT-guided decision making had on average a shorter duration of antibiotics treatment than neonates treated following standard care (Table 2). However, mean duration of hospitalization differed between countries, with slightly shorter hospital stay for the standard care group in all countries combined ( $2 \mathrm{~h}$ ), mainly caused by a shorter duration of hospitalization in the Czech Republic (96 h). The Netherlands, Switzerland and Canada, on the other hand, had on average a shorter duration of hospitalization for the PCT-guided decision-making group than for the standard care group (minus 1,16 and $5 \mathrm{~h}$ respectively). Comparing countries, it was found that Switzerland had on average the longest duration of hospitalization, both for the PCT-guided decision-making group and for the standard care group. The Netherlands had on average the shortest duration of hospitalization for the PCTguided decision-making group, whereas the Czech Republic had the shortest duration of hospitalization for standard care. For both PCT-guided decision making and standard care, duration of antibiotics treatment was on average shortest in Canada. Longest duration of antibiotic treatment was found for the Czech Republic (PCT-guided decision making) and Switzerland (standard care).

\section{Costs}

Overall, it was found that costs of PCT-guided decision making were higher than costs of standard care, but not significantly ( $€ 3649$ vs. $€ 3616, P=0.240$ ) (Table 3 ). Subgroup analysis showed that costs were significantly different between countries. However, costs were not significantly different between PCT-guided decision making and standard care within all countries, except for the Czech Republic, where standard care had significantly lower costs than PCT-guided decision making $(€ 1242$ versus $€ 2328, P<0.001$ ).

Considering all countries together, subgroup analysis of costs per risk category showed on average significantly lower costs for PCT-guided decision making compared to standard care in risk category 'infection unlikely' $(P=0.041)$ (Fig. 2). Costs for risk category 'infection possible' were found to be slightly higher for the PCT-guided decision-making group, but not significantly. Considering risk categories within countries, it was found that in both the Netherlands, Switzerland and the Czech Republic costs were significantly lower for PCT-guided decision making in risk category 'infection unlikely'. In addition, for the Czech Republic significantly higher costs of PCT-guided decision making were found for risk group 'infection possible.' No significant differences between PCT-guided decision making and standard care were found for Canada.

Late preterm neonates (gestational age $<37$ weeks) had significantly higher costs compared to term neonates (gestational age $\geq 37$ weeks), both for PCT-guided decision making ( $€ 6436$ vs. $€ 3081, P<0.001)$ and for standard care $(€ 6098$ vs. $€ 2986, P<0.001)$, considering all countries together. Similar significant results were found for the Netherlands and Switzerland, whereas for Canada and the Czech Republic the difference was not significant. Subgroup analyses of gestational age groups within countries showed a significant difference between PCTguided decision making and standard care for Switzerland in gestational age $\geq 37$ weeks group only, and for the Czech Republic in both gestational age $<37$ weeks and $\geq 37$ weeks.

Considering total health care use, it was found that all health care use, except for hospital hours, was significantly different $(P<0.05)$ between the two treatment groups (Table 4). The main contributor to health care costs was costs of hospitalization, both for PCT-guided decision making and standard care (Fig. 2).

\section{Sensitivity analysis}

Differences in mean health care costs between the PCTguided decision-making group and the standard care group remained insignificant, using both the minimum 


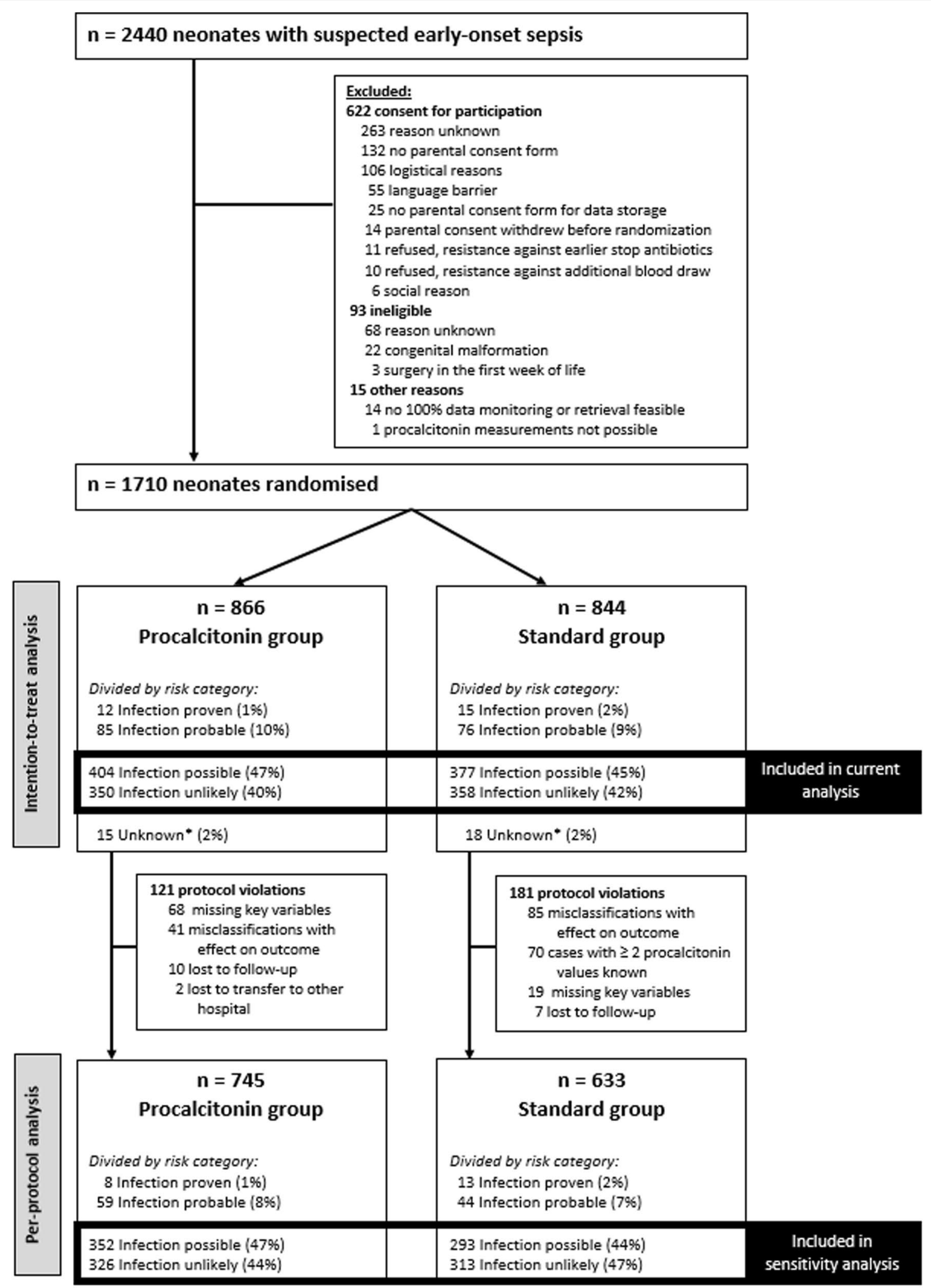

Fig. 1 Flowchart. *Unknown are neonates with missing key variables 
Table 1 Patient characteristics of intention to treat population

\begin{tabular}{|c|c|c|c|}
\hline & $\begin{array}{l}\text { PCT-guided } \\
\text { decision } \\
\text { making } \\
n=754\end{array}$ & $\begin{array}{l}\text { Standard care } \\
n=735\end{array}$ & $P$ value \\
\hline Male sex & $452(59.9 \%)$ & $428(58.2 \%)$ & 0.501 \\
\hline Country & & & 0.440 \\
\hline Netherlands & $439(58.2 \%)$ & $442(60.1 \%)$ & \\
\hline Switzerland & $124(16.4 \%)$ & $111(15.1 \%)$ & \\
\hline Canada & $152(20.2 \%)$ & $155(21.1 \%)$ & \\
\hline Czech Republic & $39(5.2 \%)$ & $27(3.7 \%)$ & \\
\hline Gestational age (in weeks) & $38.5(2.2)$ & $38.5(2.2)$ & 0.731 \\
\hline Birth weight in kg & $3.3(0.66)$ & $3.4(0.62)$ & 0.346 \\
\hline Delivery way & & & 0.051 \\
\hline $\begin{array}{l}\text { Spontaneous vaginal } \\
\text { delivery }\end{array}$ & $375(49.7 \%)$ & $331(45.0 \%)$ & \\
\hline $\begin{array}{l}\text { Vacuum/forceps } \\
\text { delivery }\end{array}$ & $114(15.1 \%)$ & $133(18.1 \%)$ & \\
\hline C-section & $263(34.9 \%)$ & $270(36.7 \%)$ & \\
\hline Missing & $2(0.3 \%)$ & $1(0.1 \%)$ & \\
\hline Arterial cord pH & $7.2(0.1)$ & $7.2(0.1)$ & 0.371 \\
\hline Missing & $135(17.9 \%)$ & $142(19.3 \%)$ & \\
\hline \multicolumn{4}{|l|}{ Apgar score } \\
\hline 1 min postpartum & $7.4(2.2)$ & $7.3(2.3)$ & 0.378 \\
\hline Missing & $1(0.1 \%)$ & $6(0.8 \%)$ & \\
\hline 5 min postpartum & $8.6(1.6)$ & $8.5(1.7)$ & 0.245 \\
\hline Missing & $0(-)$ & $8(1.1 \%)$ & \\
\hline 10 min postpartum & $8.9(1.3)$ & $8.9(1.3)$ & 0.567 \\
\hline Missing & $276(36.6 \%)$ & $255(34.7 \%)$ & \\
\hline Risk category & & & 0.377 \\
\hline $\begin{array}{l}\text { Infection possible } \\
\text { (medium risk) }\end{array}$ & $404(53.6 \%)$ & $377(51.3 \%)$ & \\
\hline $\begin{array}{l}\text { Infection unlikely (low } \\
\text { risk) }\end{array}$ & $350(46.4 \%)$ & $358(48.7 \%)$ & \\
\hline
\end{tabular}

The italics were used to represent missing values per variable Data are expressed as mean (SD) or $n(\%)$

$S D$ standard deviation

${ }^{*}$ Significant at a $5 \%$ level $(P<0.05)$ and maximum price of a PCT-test (Table 5). However, for risk category 'infection unlikely', it was found that the difference in costs between the two groups was no longer significant when using the maximum price for a PCTtest, whereas it was significant when using the minimum price. For the Netherlands, it was found that the difference in costs was significant when using the minimum price for PCT-testing, whereas it was no longer significant when the maximum price for PCT-testing was used. For the Czech Republic, the difference in costs remained significant using both the minimum and maximum price.

The sensitivity analyses excluding additional days of hospitalization due to SAEs showed that mean duration of hospitalization was significantly $(P<0.001)$ shorter for PCT-guided decision making than for standard care (Table 6). In addition, mean total health care costs were significantly $(P<0.001)$ lower for PCT-guided decision making compared to standard care.

Sensitivity analyses of the per protocol population, comparing duration of hospitalization including hospitalization due to SAEs and total costs of health care including SAEs, showed a significant difference in duration of hospitalization between PCT-guided decision making and standard care (162 h versus $159 \mathrm{~h}, P=0.035)$. However, total costs of health care were not significantly different between PCT-guided decision making and standard care.

\section{Discussion \\ Main findings}

This study compared medical costs of neonates born after 34 weeks of gestational age, who had suspected low risk of EOS in the first $72 \mathrm{~h}$ of life and required antibiotic therapy, between an intervention (PCT-guided decisionmaking) and control group (standard care according to local policy). Neonates were considered to have a low risk when they were classified in either risk category 'infection possible' or 'infection unlikely.' Mean total costs of health care were not significantly different between the

Table 2 Mean $[\mathrm{IQR}]$ duration of hospitalization and antibiotic treatment in hours per country

\begin{tabular}{|c|c|c|c|c|}
\hline & \multicolumn{2}{|c|}{ PCT-guided decision making $(n=754)$} & \multicolumn{2}{|l|}{ Standard care $(n=735)$} \\
\hline & $\begin{array}{l}\text { Duration of hospitalization } \\
\text { mean [IQR] }\end{array}$ & $\begin{array}{l}\text { Duration of antibiotic } \\
\text { treatment mean [IQR] }\end{array}$ & $\begin{array}{l}\text { Duration of hospitalization } \\
\text { mean [IQR] }\end{array}$ & $\begin{array}{l}\text { Duration of antibiotic } \\
\text { treatment mean [IQR] }\end{array}$ \\
\hline All countries & $164[69,184]$ & $74[32,114]$ & $162[79,183]$ & $87[49,120]$ \\
\hline The Netherlands & $134[63,165]$ & $72[30,120]$ & $135[71,166]$ & $88[51,130]$ \\
\hline Switzerland & $235[120,303]$ & $86[42,120]$ & $251[143,289]$ & $101[60,121]$ \\
\hline Canada & $181[81,192]$ & $67[30,78]$ & $186[87,218]$ & $74[47,71]$ \\
\hline Czech Republic & $207[116,288]$ & $91[36,133]$ & $111[69,146]$ & $96[58,132]$ \\
\hline
\end{tabular}

Data are expressed in hours as mean + IQR (interquartile range)

Antibiotic treatment includes both IV and oral treatment 


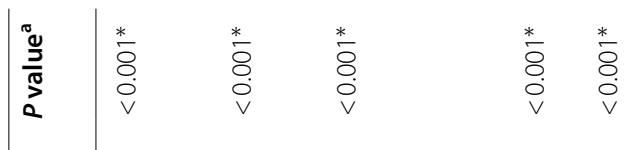

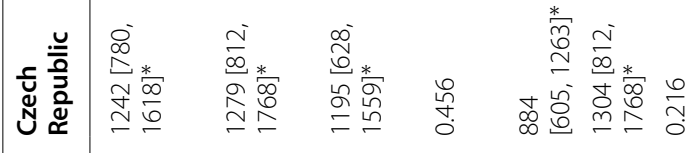

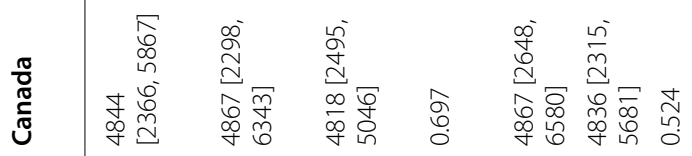

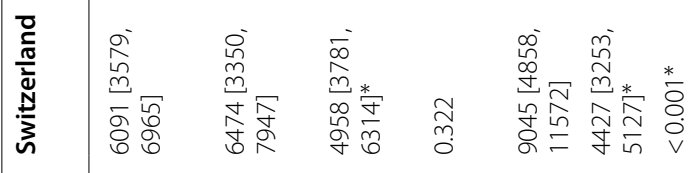

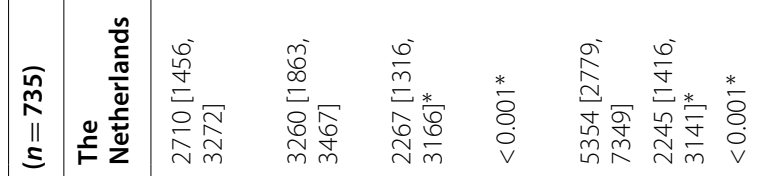

थै

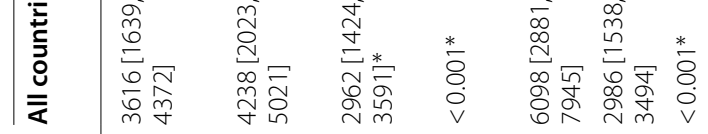

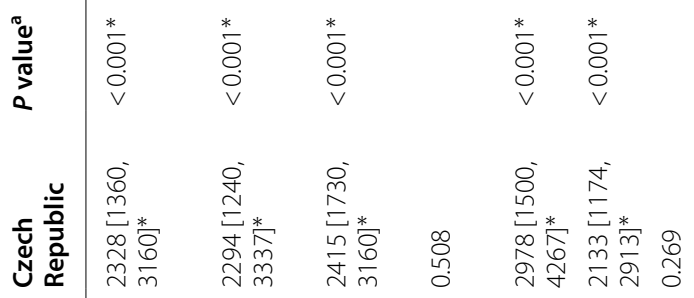

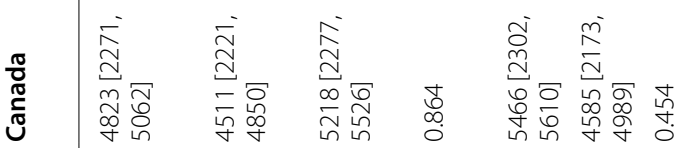




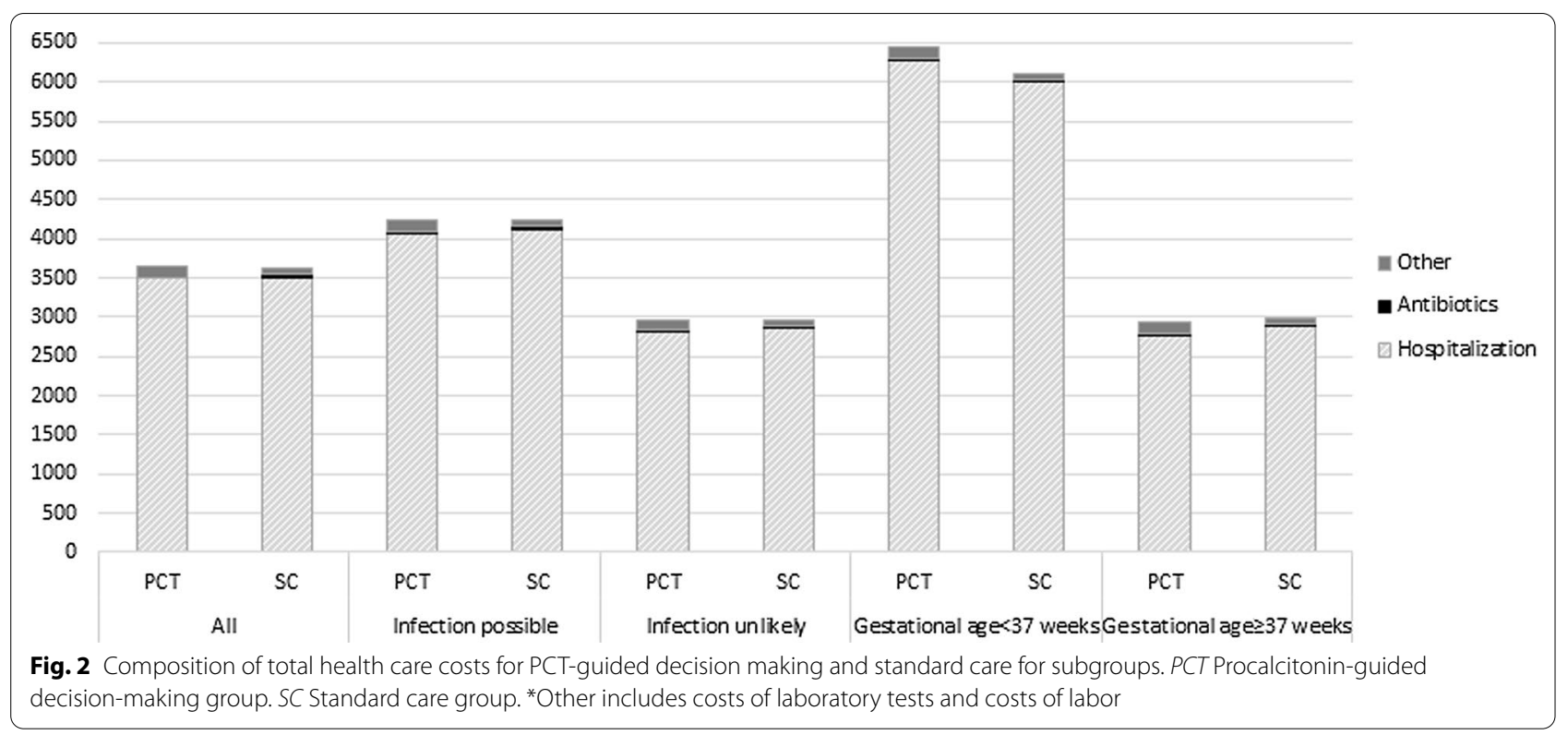

Table 4 Resource use and costs of health care (2015€) per patient for the population

\begin{tabular}{|c|c|c|c|c|c|}
\hline & \multicolumn{2}{|c|}{ PCT-guided decision making $(n=754)$} & \multicolumn{2}{|l|}{ Standard care $(n=735)$} & \multirow[t]{2}{*}{$P$ value $^{\mathrm{a}}$} \\
\hline & Resource use (number) & Costs (euro) & Resource use (number) & Costs (euro) & \\
\hline PCT number of tests & $3[2,4]$ & $63[39,79]$ & NA & NA & $<0.001^{*}$ \\
\hline CRP number of tests & $4[3,4]$ & $15[12,16]$ & $3[3,4]$ & $14[10,16]$ & $0.001^{*}$ \\
\hline Hospital hours & $164[69,184]$ & $3489[1377,3906]$ & $162[79,183]$ & $3511[1541,4215]$ & 0.060 \\
\hline Antibiotic doses & $10[5,12]$ & $22[9,25]$ & $12[7,14]$ & $26[13,29]$ & $<0.001^{*}$ \\
\hline Labor costs & - & $61[43,76]$ & - & $66[52,79]$ & $<0.001^{*}$ \\
\hline Total costs & - & $3649[1496,4091]$ & - & $3616[1639,4372]$ & 0.240 \\
\hline
\end{tabular}

Data are expressed as mean + IQR (interquartile range)

Costs are expressed in $€$ corrected for inflation for the year 2015

a $P$ value for the difference in costs between PCT-guided decision making and standard care

${ }^{*}$ Significant at $5 \%$ level $(P<0.05)$

$\mathrm{NA}=$ not applicable

PCT-guided decision-making group and the standard care group. Between countries, there was a difference in the mean duration of hospitalization and antibiotic treatment, which probably can be explained by broad diversity in local policy [16]. In risk category 'infection possible', costs of health care were found to be (not significantly) higher for PCT-guided decision making. This was an unexpected finding, as duration of hospitalization was expected to be shorter in the PCT-guided decision-making group. Considering the sensitivity analyses excluding SAEs, it was found that duration of hospitalization and total costs were significantly shorter and lower for PCTguided decision making, compared to standard care. This indicates that neonates in the PCT-guided decision-making group had higher costs and longer hospitalization than neonates in the standard care group due to SAEs. Although exclusion of SAEs reflects the true potential of the intervention on reducing costs and hospitalization, in clinical practice additional days due to prematurity related problems will remain. However, reducing intravenous antibiotic therapy will not only benefit the duration of treatment/hospitalization, but is also important for improving mother and child bonding, reducing the use of monitor facilities, reducing additional procedures as IV-catheters and moreover reducing the possible alterations microbiome that could possibly lead to microbial resistance. For risk category 'infection unlikely', on the other hand, health care costs were significantly lower for PCT-guided decision making. This was an expected result as treatment in this risk category was affected by 
Table 5 Costs of care with lowest and highest price for PCT-test (2015€)

\begin{tabular}{|c|c|c|c|c|c|c|}
\hline & \multicolumn{2}{|c|}{ PCT-guided decision making $(n=754)$} & \multicolumn{2}{|c|}{ Standard care $(n=735)$} & \multicolumn{2}{|l|}{$P$ value } \\
\hline & Min. price & Max. price & Min. price & Max. price & Min. price & Max. price \\
\hline All patients (in total) & $3630[1479,4073]$ & $3695[1533,4137]$ & $3616[1639,4372]$ & $3616[1639,4372]$ & 0.154 & 0.534 \\
\hline \multicolumn{7}{|l|}{ Per risk category } \\
\hline Infection possible (medium risk) & $4226[1964,4798]$ & $4296[2033,4916]$ & $4238[2023,5021]$ & $4238[2023,5021]$ & 0.648 & 0.938 \\
\hline Infection unlikely (low risk) & $2942[1281,3370]$ & $3001[1330,3441]$ & $2962[1424,3591]$ & $2962[1424,3591]$ & $0.023^{*}$ & 0.112 \\
\hline \multicolumn{7}{|l|}{ Per country } \\
\hline Netherlands & $2731[1296,3292]$ & $2793[1352,3372]$ & $2710[1456,3272]$ & $2710[1456,3272]$ & $0.048^{*}$ & 0.288 \\
\hline Switzerland & $5783[3044,7388]$ & $5884[3137,7485]$ & $6091[3579,6965]$ & $6091[3579,6965]$ & 0.190 & 0.330 \\
\hline Canada & $4809[2259,5051]$ & $4855[2299,5119]$ & $4844[2366,5867]$ & $4844[2366,5867]$ & 0.102 & 0.151 \\
\hline Czech Republic & $2312[1343,3146]$ & $2366[1401,3193]$ & $1242[780,1618]$ & $1242[780,1618]$ & $<0.001^{*}$ & $<0.001^{*}$ \\
\hline
\end{tabular}

Data are expressed as mean + IQR (interquartile range).

Min minimum, Max maximum, $I Q R$ interquartile range

*Significant at $5 \%$ level $(P<0.05)$

Costs are expressed in $€$ corrected for inflation for the year 2015

Table 6 Mean duration of hospitalization and costs of care excluding duration of hospitalization due to SAEs, and excluding neonates with protocol violations

\begin{tabular}{llll}
\hline & PCT-guided decision making & Standard care & $P$ value \\
\hline Excluding SAEs & & & $<0.001^{*}$ \\
Duration of hospitalization in hours & $97[48,139]$ & $107[64,146]$ & $<0.001^{*}$ \\
Total costs of health care in euro & $2166[1132,3084]$ & $2349[1387,3087]$ & \\
Excluding neonates with protocol violations & $162[67,181]$ & $159[77,181]$ & $0.035^{*}$ \\
Duration of hospitalization in hours & $3735[1496,4159]$ & $3578[1636,4293]$ & 0.412 \\
Total costs of health care in euro & &
\end{tabular}

Data are expressed as mean + IQR (interquartile range)

SAE Serious adverse event

*Significant at $5 \%$ level $(P<0.05)$

Costs are expressed in $€$ corrected for inflation for the year 2015

the intervention. However, sensitivity analyses showed that a higher price of PCT-testing resulted in a nonsignificant difference. Therefore, a significant cost reduction for PCT-guided decision making depends on the price of PCT-testing. The price difference in PCT-tests between laboratories is mainly caused by variation in number of PCT-tests that are performed in a hospital. The more PCT-tests a hospital performs, the lower the costs per test can be.

\section{Comparison to previous studies}

To the best of our knowledge, there have been no costeffectiveness studies on PCT-guided decision making in children. Several studies have been performed on costs of PCT-guided decision making in adults with sepsis, which found significant health care costs reductions when PCT-guided decision making was applied $[8,9,11$, $12]$. One study, however, found no significant difference in health care costs during initial hospitalization in critically ill adults, which is in line with our findings [10]. The subgroup analyses of our study, however, showed a significant reduction in costs for risk category 'infection unlikely' when PCT-guided decision making was applied.

A previous study on PCT-guided decision making found that antibiotic use and adverse events related to antibiotic use could be significantly reduced in children aged 1 month to 14 years old with pneumonia [17]. The significant reduction in antibiotics use was in line with our findings, and led to lower costs of antibiotics. Another study in a similar population (children aged 1 month to 18 years) with LRTI also found a significant reduction in duration of antibiotic treatment with PCTguided decision making [18].

\section{Strengths and limitations}

A major strength of this study is that because of the pragmatic approach of the original study, with a large number 
of neonates in different countries, the results of this costminimization analysis can be directly translated into current clinical practice in different clinical settings within the Netherlands. In addition, it provides an estimation of costs in other high-income countries. Even though there is variation in health care costs within these high-income countries, this study still shows that PCT-guided decision making remains to be cost-effective in the lowest risk category. However, cost estimations for other countries should be interpreted with caution, as the resource prices were calculated using the Dutch price, corrected for PPP for GDP.

One major limitation of our study is that duration of stay at the hospital is possibly biased due to SAEs. The majority of the SAEs resulted in prolonged hospitalization due to prematurity related causes, like feeding intolerance or hyperbilirubinemia [7]. Therefore, duration of hospital stay due to sepsis is overestimated for these patients. However, the sensitivity analysis, using duration of hospitalization excluding SAEs, showed the real potential of PCT-guided decision making.

Furthermore, costs in this study only represent health care costs. Additional societal costs, such as costs from missed work days of the care-givers of the neonates, were not taken into account. Since not all countries provide extensive parental leave, especially for the partner, this could induce additional costs.

\section{Implications for practice}

Applying antibiotic stewardship in neonates suspected with EOS has several practical benefits, besides reducing the duration of antibiotic therapy and diminishing long-term effects on a neonates health. Firstly, there will be less use of cardiorespiratory monitoring, which is particularly interesting in hospitals with limited monitoring capacities. Subsequently, less antibiotic treatment also implicates a reduction in use of personnel hours: from less need to prepare intravenous medication, to less attempts to gain intravenous access to administer antibiotic therapy. Due to the COVID pandemic, the threshold to implement PCT in laboratories is lowered. PCT-testing is now possible on almost all platforms used by the majority of the hospital laboratories. A higher use and availability of PCT-testing will also lower the cost price of procalcitonin testing, resulting in larger cost reductions.

\section{Conclusion}

In conclusion, applying PCT-guided decision making in neonates born after 34 weeks of gestational age, with a low risk of suspected EOS in the first $72 \mathrm{~h}$ of life requiring antibiotic therapy, resulted in a nonsignificant difference in total health care costs compared to standard care. A significant cost reduction was found for subgroup 'infection unlikely.' However, cost reduction is affected by both the price of PCT-testing and hospitalization due to SAEs.

\section{Abbreviations}

PCT: Procalcitonin; EOS: Early-onset sepsis; SAE: Serious adverse event; LRTI: Lower respiratory tract infection; CRF: Case record form; NL: Netherlands; $\mathrm{CH}$ : Switzerland; CA: Canada; IQR: Interquartile range.

\section{Supplementary Information}

The online version contains supplementary material available at https://doi. org/10.1186/s13054-021-03789-x.

Additional file 1: Figure S1. Risk classification and duration of antibiotic therapy using normal values of procalcitonin [7].

Additional file 2: Table S1. Overview cost prices in $2015 €$.

\section{Acknowledgements}

Not applicable.

\section{Authors' contributions}

$A G$ and WH designed the study, analyzed and interpreted the data, drafted the article and revised it critically. MS, SH, MF, FS, RT, JW, LM, RM, AD, UZ, AH, MR, MT, JG, EV, SD, JJ, SS, EV, LS, AM and RK collected data and critically revised the manuscript. AR and SP conceptualized and designed the study, interpreted the data and critically revised the manuscript. All authors critically reviewed the manuscript and approved the final manuscript as submitted and agree to be accountable for all aspects of work.

Funding

Not applicable.

Availability of data and materials

Not applicable.

\section{Declarations}

\section{Ethics approval and consent to participate}

The local institutional review board and the national ethical committee of each site approved the study, and written informed consent was obtained from the parents or guardians for all patients. The trial was registered at ClinicalTrials.gov (NCT00854932).

\section{Consent for publication}

Not applicable.

\section{Competing interests}

The authors declare that they have no competing interests.

\section{Author details}

${ }^{1}$ Department of Public Health, Erasmus MC, University Medical Center Rotterdam, P.O. Box 2040, 3000 CA Rotterdam, The Netherlands. ${ }^{2}$ Division of Paediatric Infectious Diseases \& Immunology, Department of Paediatrics, Erasmus MC University Medical Centre - Sophia Children's Hospital, Rotterdam, The Netherlands. ${ }^{3}$ Department of Paediatrics, Neonatal and Paediatric Intensive Care Unit, Children's Hospital Lucerne, Lucerne, Switzerland. ${ }^{4}$ Division of Neonatology, McMaster University Children's Hospital, Hamilton Health Sciences, Hamilton, ON, Canada. ${ }^{5}$ Neonatal Intensive Care Unit, Isala Women and Children's Centre, Isala Hospital, Zwolle, The Netherlands. ${ }^{6}$ Department of Paediatrics, Haaglanden Medical Center, 's Gravenhage, The Netherlands. ${ }^{7}$ Neonatal Unit, Department of Obstetrics and Gynaecology, Motol University Hospital, Second Medical Faculty, Charles University, Prague, Czech Republic. ${ }^{8}$ Institute of Pathological Physiology, First Medical Faculty, Charles University, Prague, Czech Republic. ${ }^{9}$ Department of Neonatology, Reinier de Graaf Gasthuis, Delft, The Netherlands. ${ }^{10}$ Department of Neonatology, Zuyderland 
Medical Centre, Heerlen, The Netherlands. " 1 Department of Neonatology, Amsterdam UMC, Vrije Universiteit Amsterdam, Amsterdam, The Netherlands. ${ }^{12}$ Department of Paediatrics, Jeroen Bosch Hospital, 's-Hertogenbosch, The Netherlands. ${ }^{13}$ Department of Paediatrics, Maxima Medical Centre, Veldhoven, The Netherlands. ${ }^{14}$ Department of Paediatrics, Kantonsspital Winterthur, Winterthur, Switzerland. ${ }^{15}$ Department of Paediatrics, Bern University Hospital, Inselspital, University of Bern, Bern, Switzerland. ${ }^{16}$ Paediatric Critical Care Research Group, Mater Research Institute, University of Queensland, Brisbane, QLD, Australia. ${ }^{17}$ Paediatric Intensive Care Unit, Lady Cilento Children's Hospital, Brisbane, QLD, Australia. ${ }^{18}$ Department of Neonatology, Albert Schweitzer Hospital, Dordrecht, The Netherlands. ${ }^{19}$ Department of Neonatology, Sint Franciscus Gasthuis, Rotterdam, The Netherlands. ${ }^{20}$ Department of Neonatology, St. Josephs Healthcare, Hamilton Health Sciences, Hamilton, ON, Canada. ${ }^{21}$ Department of Paediatrics, Stadtspital Triemli, Zürich, Switzerland. ${ }^{22}$ Division of Neonatology, Erasmus MC University Medical Centre-Sophia Children's Hospital, Rotterdam, The Netherlands. ${ }^{23}$ Julius Training General Practitioner, University Medical Centre Utrecht, Utrecht, The Netherlands.

Received: 3 August 2021 Accepted: 8 October 2021

Published online: 20 October 2021

\section{References}

1. Vergnano S, Menson E, Kennea N, Embleton N, Russell AB, Watts T, et al. Neonatal infections in England: the NeonIN surveillance network. Arch Dis Child Fetal Neonatal Ed. 2011;96(1):F9-14.

2. Zimmermann $P$, Curtis N. Effect of intrapartum antibiotics on the intestinal microbiota of infants: a systematic review. Arch Dis Child Fetal Neonatal Ed. 2020;105(2):201-8.

3. Afroza S. Neonatal sepsis - a global problem: an overview. Mymensingh Med J. 2006;15(1):108-14.

4. Assicot M, Gendrel D, Carsin H, Raymond J, Guilbaud J, Bohuon C. High serum procalcitonin concentrations in patients with sepsis and infection. Lancet. 1993;341(8844):515-8.

5. van Rossum AM, Wulkan RW, Oudesluys-Murphy AM. Procalcitonin as an early marker of infection in neonates and children. Lancet Infect Dis. 2004;4(10):620-30.

6. Vouloumanou EK, Plessa E, Karageorgopoulos DE, Mantadakis E, Falagas ME. Serum procalcitonin as a diagnostic marker for neonatal sepsis: a systematic review and meta-analysis. Intensive Care Med. 2011;37(5):747-62.

7. Stocker M, van Herk W, El Helou S, Dutta S, Fontana MS, Schuerman F, et al. Procalcitonin-guided decision making for duration of antibiotic therapy in neonates with suspected early-onset sepsis: a multicentre, randomised controlled trial (NeoPIns). Lancet. 2017;390(10097):871-81.

8. Balk RA, Kadri SS, Cao Z, Robinson SB, Lipkin C, Bozzette SA. Effect of procalcitonin testing on health-care utilization and costs in critically III patients in the United States. Chest. 2017;151(1):23-33.
9. Schroeder S, Hochreiter M, Koehler T, Schweiger AM, Bein B, Keck FS, et al. Procalcitonin (PCT)-guided algorithm reduces length of antibiotic treatment in surgical intensive care patients with severe sepsis: results of a prospective randomized study. Langenbecks Arch Surg. 2009;394(2):221-6.

10. Kip MMA, van Oers JA, Shajiei A, Beishuizen A, Berghuis AMS, Girbes $A R$, et al. Cost-effectiveness of procalcitonin testing to guide antibiotic treatment duration in critically ill patients: results from a randomised controlled multicentre trial in the Netherlands. Crit Care. 2018;22(1):293.

11. Mewes JC, Pulia MS, Mansour MK, Broyles MR, Nguyen HB, Steuten LM. The cost impact of PCT-guided antibiotic stewardship versus usual care for hospitalised patients with suspected sepsis or lower respiratory tract infections in the US: A health economic model analysis. PLoS One. 2019;14(4):e0214222.

12. Voermans AM, Mewes JC, Broyles MR, Steuten LMG. Cost-Effectiveness Analysis of a Procalcitonin-Guided Decision Algorithm for Antibiotic Stewardship Using Real-World U.S. Hospital Data. OMICS. 2019;23(10):508-15.

13. Stocker M, Hop WC, van Rossum AM. Neonatal Procalcitonin Intervention Study (NeoPInS): effect of Procalcitonin-guided decision making on duration of antibiotic therapy in suspected neonatal early-onset sepsis: a multi-centre randomized superiority and non-inferiority Intervention Study. BMC Pediatr. 2010;10:89.

14. Hakkaart-van Roijen L, van der Linden N, Bouwmans C, Kanters T, Swan Tan S. Kostenhandleiding: Methodologie van kostenonderzoek en referentieprijzen voor economische evaluaties in de gezondheidszorg. Rotterdam: Institute for Medical Technology Assessment; Erasmus Universiteit Rotterdam; 2015.

15. Zorginstituut Nederland. Farmacotherapeutisch Kompas 2020 [Available from: http://www.farmacotherapeutischkompas.nl.

16. van Herk W, el Helou S, Janota J, Hagmann C, Klingenberg C, Staub E, et al. Variation in current management of term and late-preterm neonates at risk for early-onset sepsis: an international survey and review of guidelines. Pediatr Infect Dis J. 2016;35(5):494-500.

17. Esposito S, Tagliabue C, Picciolli I, Semino M, Sabatini C, Consolo S, et al. Procalcitonin measurements for guiding antibiotic treatment in pediatric pneumonia. Respir Med. 2011;105(12):1939-45.

18. Baer G, Baumann P, Buettcher M, Heininger U, Berthet G, Schafer J, et al. Procalcitonin guidance to reduce antibiotic treatment of lower respiratory tract infection in children and adolescents (ProPAED): a randomized controlled trial. PLoS ONE. 2013;8(8):e68419.

\section{Publisher's Note}

Springer Nature remains neutral with regard to jurisdictional claims in published maps and institutional affiliations.

\footnotetext{
Ready to submit your research? Choose BMC and benefit from:

- fast, convenient online submission

- thorough peer review by experienced researchers in your field

- rapid publication on acceptance

- support for research data, including large and complex data types

- gold Open Access which fosters wider collaboration and increased citations

- maximum visibility for your research: over $100 \mathrm{M}$ website views per year
}

At $\mathrm{BMC}$, research is always in progress.

Learn more biomedcentral.com/submissions 